\title{
COSMOLOGICAL BACKGROUND OF GRAVITATIONAL WAVES
}

\author{
MAREK DEMIAŃSKI \\ Institute of Theoretical Physics, University of Warsaw, \\ Hoża 69, 00-681 Warsaw, Poland \\ E-mail: mde@fuw.edu.pl
}

Several different astrophysical sources of gravitational radiation have been discussed at this workshop. I would like to briefly review expected properties of the cosmological background of gravitational waves. Let me however begin with a general introduction. After formulating the general theory of relativity Albert Einstein showed that in the weak field approximation equations of general relativity possess wave like solutions. Let the background spacetime be the Minkowski spacetime parametrized by the Cartesian coordinates, then

$$
\eta_{\alpha \beta}=\operatorname{Diag}\|+1,-1,-1,-1\|,
$$

where $\alpha, \beta=0,1, \cdots, 3$. Slightly perturbed Minkowski spacetime will be described by the metric tensor

$$
g_{\alpha \beta}\left(x^{\mu}\right)=\eta_{\alpha \beta}+h_{\alpha \beta}\left(x^{\mu}\right),
$$

where $h_{\alpha \beta}(x) \ll 1$ represent small perturbations. Using the standard definition of the Ricci tensor and keeping only terms linear in $h$ we obtain

$$
R_{\alpha \beta}=\frac{1}{2}\left(-\eta^{\gamma \delta} h_{\alpha \beta, \gamma \delta}+h_{\alpha}^{\gamma}{ }_{\gamma \beta}+h_{\beta}^{\gamma},_{\gamma \alpha}-h_{, \alpha \beta}\right),
$$

where $h=h_{\alpha \beta} \eta^{\alpha \beta}, h_{\alpha}^{\gamma}=\eta^{\gamma \beta} h_{\alpha \beta}$. Using still existing coordinate freedom $x^{\mu} \rightarrow x^{\mu}+$ $\xi^{\mu}(x)$, where $\xi^{\mu}$ are assumed to be small, we can impose the following gauge conditions

$$
h_{\alpha, \beta}^{\beta}=0 ; \quad h=h_{\alpha \beta} \eta^{\alpha \beta}=0,
$$

and require that

$$
h_{\alpha \beta} u^{\beta}=0, \quad \text { for } \quad u_{\alpha} u^{\alpha}=1 .
$$

This gauge is called TT-gauge (traceless-transverse gauge). In this gauge the Einstein

1991 Mathematics Subject Classification: 83C35, 83F05.

The paper is in final form and no version of it will be published elsewhere. 
field equations in vacuum reduce to

$$
\eta^{\gamma \delta} h_{\alpha \beta, \gamma \delta}=0 \text {. }
$$

For a plane wave propagating in the $z$ direction we obtain the following two independent solutions

$$
\begin{gathered}
h_{+}=h_{x x}=-h_{y y}=\operatorname{Re}\left\{A_{+} e^{-i \omega\left(t-\frac{z}{c}\right)}\right\}, \\
h_{\times}=h_{x y}=h_{y x}=\operatorname{Re}\left\{A_{\times} e^{-i \omega\left(t-\frac{z}{c}\right)}\right\},
\end{gathered}
$$

where $A_{+}$and $A_{\times}$denote amplitudes of the two waves.

It took much longer to study and understand properties of gravitational waves produced by a bounded distribution of matter. Only late in 50s mostly due to the work of Petrov, Pirani, Trautman, Bondi, Sachs, Penrose, and many others, it became clear that gravitational waves in an asymptotically flat space are linked to asymptotic properties of the Riemann tensor and its algebraic classification. Riemann tensor produced by a bounded distribution of matter in an asymptotically flat space can be, far from the sources, expanded along the future null cone and written in the form

$$
R_{\alpha \beta \gamma \delta}=\frac{N_{\alpha \beta \gamma \delta}}{r}+\frac{I I I_{\alpha \beta \gamma \delta}}{r^{2}}+\frac{I I_{\alpha \beta \gamma \delta}}{r^{3}}+\cdots
$$

where $r$ is the affine parameter along outgoing null rays spanning the future null cone, and

$$
\begin{gathered}
N_{\alpha \beta \gamma \delta} k^{\delta}=0, \\
I I I_{\alpha \beta \gamma[\delta} k_{\sigma]} k^{\gamma}=0, \\
I I_{\alpha \beta \gamma[\delta} k_{\sigma]} k^{\beta} k^{\gamma}=0,
\end{gathered}
$$

where $k_{\alpha} \sim u_{, \alpha}$, and $u=$ const describes the future null cone.

Study of gravitational waves in cosmological spacetimes was initiated early in 40 s by E. M. Lifshitz [7] who in his pioneering paper published in 1946 discussed the general perturbations of the Friedman models. Among the three basic perturbations he included tensor perturbations describing gravitational waves. This analysis was later repeated by many authors. Properties of gravitational waves propagating in the Friedman cosmological spacetimes were discussed by L. P. Grishchuk [5]. A few years later L. Ford and L. Parker [4] described the quantum process of graviton creation.

Let me present basic results concerning gravitational waves obtained by Lifshitz and Grishchuk. Let us consider a general background spacetime with the line element

$$
d s^{2}=\gamma_{\alpha \beta} d x^{\alpha} d x^{\beta},
$$

and introduce small perturbations by changing the background metric to

$$
g_{\alpha \beta}=\gamma_{\alpha \beta}+h_{\alpha \beta},
$$

where $\left|h_{\alpha \beta}\right| \ll 1$. It turns out that the equations for small perturbations can be written down in a simpler form if instead of $h_{\alpha \beta}$ we use a new quantity $\psi_{\alpha \beta}$ defined by

$$
\psi_{\alpha \beta}=h_{\alpha \beta}-\frac{1}{2} \gamma_{\alpha \beta} h,
$$

where $h=h_{\alpha \beta} \gamma^{\alpha \beta}$. 
The division of the spacetime into the background and small perturbations does not fix the coordinate system uniquely. We still have the freedom to perform coordinate transformations of the form $x^{\mu} \rightarrow x^{\mu}+\xi^{\mu}(x)$. Using this freedom we can set

$$
\psi_{; \beta}^{\alpha \beta}=0 \text {, }
$$

where the covariant derivative is taken with respect to the background metric. With this additional restriction on $\psi$ the Einstein field equations linearized with respect to $\psi_{\alpha \beta}$ lead to

$$
\psi_{\mu \nu ; \alpha}{ }^{\alpha}-2 R_{\alpha \mu \nu \beta}^{(0)} \psi^{\alpha \beta}+\psi_{\mu \alpha} R_{\nu}^{(0)}{ }_{\nu}^{\alpha}+\psi_{\alpha \nu} R_{\mu}^{(0)}{ }_{\mu}^{\alpha}=T_{\mu \nu}^{(1)}
$$

where $R_{\alpha \beta \mu \nu}^{(0)}$ is the background Riemann tensor, $R_{\alpha \beta}^{(0)}$ is the background Ricci tensor, and $T_{\alpha \beta}^{(1)}$ describes small perturbations of the background matter. The remaining coordinate freedom $x^{\mu} \rightarrow x^{\mu}+\xi^{\mu}(x)$ is restricted by

$$
\xi_{\mu ; \alpha}^{; \alpha}+\xi^{\alpha} R_{\alpha \mu}^{(0)}=0 .
$$

In the vacuum spacetime the remaining coordinate freedom can be used to set $\psi=$ $\gamma^{\alpha \beta} \psi_{\alpha \beta}=0$ and the equations governing the small perturbations assume the form

$$
h_{\mu \nu ; \alpha} ; \alpha-2 R_{\alpha \mu \nu \beta}^{(0)} h^{\alpha \beta}=0,
$$

where now $h_{\alpha \beta}$ is restricted by $h_{\mu}{ }^{\nu}{ }_{; \nu}=0$ and $h=h_{\alpha \beta} \gamma^{\alpha \beta}=0$.

The equation (15) can be simplified when we consider the Friedman spacetime as the background. In this case as in the case of the Minkowski background it is possible to impose the TT-gauge and we have

$$
\psi_{; \nu}^{\mu \nu}=0, \quad \psi=\psi_{\mu \nu} \gamma^{\mu \nu}=0, \quad h_{\mu \nu} u^{\nu}=0, \quad u_{\mu} u^{\mu}=1 .
$$

In what follows we discuss only the case of the flat $(\mathrm{K}=0)$ Friedman model with the line element of the form

$$
d s^{2}=c^{2} d t^{2}-R^{2}(t)\left(d x^{2}+d y^{2}+d z^{2}\right) .
$$

Following Lifshitz [7] we introduce the conformal time $\eta=\int d t / R(t)$ and transform the line element into conformally flat form

$$
d s^{2}=R^{2}(\eta)\left(d \eta^{2}-d x^{2}-d y^{2}-d z^{2}\right) .
$$

The equation describing propagation of gravitational waves on the Friedman background reduces to

$$
h_{i}^{k^{\prime \prime}}+2 \frac{R^{\prime}}{R} h_{i}^{k^{\prime}}+R^{2} \delta^{l m} h_{i, l, m}^{k}=0,
$$

where ' denotes differentiation with respect to the conformal time $\eta$. Let $G_{i}^{k}$ be a tensor eigenfunction of the Laplace operator, so

$$
\triangle G_{l}^{m}=k^{2} G_{l}^{m} .
$$

Writing $h_{i}^{k}$ in the form

$$
h_{i}^{k}=\frac{\mu}{R} G_{i}^{k}
$$

we finally obtain

$$
\mu^{\prime \prime}+\mu\left(k^{2}-\frac{R^{\prime \prime}}{R}\right)=0,
$$


where $k$ can be interpreted as the wavenumber and it is related to the wavelength by $k=2 \pi R / \lambda$.

This equation derived for the first time by Grishchuk [5] describes evolution of the amplitude of a gravitational wave propagating on the Friedman background. As it is apparent from (23), due to expansion of the universe, amplitude of the wave decreases inversely proportionally to the scale factor while its wavelength increases proportionally to $\mathrm{R}$ (adiabatic damping).

During the radiation dominated phase of evolution of the universe $R(t) \sim t^{1 / 2}$, so $R(\eta) \sim \eta$ and therefore the potential term in (24) is zero. When matter determines the expansion rate of the universe $R^{\prime \prime} \neq 0$ and the potential term appears in equation (24). Grishchuk [6] noticed that when $R^{\prime \prime} \neq 0$ process similar to the parametric amplification can create gravitons. However in order to estimate the present energy density of background gravitational waves and their spectrum it is necessary to specify the initial (primordial) density of gravitational waves and their spectrum. This information was lacking in the standard Big Bang cosmology. Situation has changed with invention of the inflationary scenario of the early evolution of the universe.

By now several different models of inflation have been proposed. The most economical one was invented by Andrei Linde [8], and it is now called chaotic inflation. Chaotic inflation is driven by a massive scalar field. In a patch of the early universe where the scalar field is sufficiently homogeneous its evolution is described by

$$
\ddot{\phi}+3 H \dot{\phi}+\frac{\partial V(\phi)}{\partial \phi}=0
$$

where $H=\dot{R} / R$ is the Hubble constant, and $V(\phi)$ is the potential of the scalar field. When the energy density of the scalar field determines the expansion rate of the universe the main Friedman equation reads

$$
H^{2}=\frac{\dot{R}^{2}}{R^{2}}=\frac{8 \pi}{3 m_{P l}^{2}}\left(\frac{1}{2} \dot{\phi}^{2}+V(\phi)\right)-\frac{K}{R^{2}},
$$

where $\mathrm{K}=+1,0$ or -1 . Equations (25) and (26) form a set of two coupled equations and when the potential $V(\phi)=\frac{1}{2} m^{2} \phi^{2}$ and the scalar field is initially sufficiently excited the general solution quickly assumes the following form

$$
\begin{gathered}
\phi(t)=\phi_{0}-\frac{m m_{P l}}{\sqrt{12 \pi}} t \\
R(t)=R_{0} \exp \left[\frac{2 \pi}{m_{P l}^{2}}\left(\phi_{0}^{2}-\phi^{2}(t)\right)\right] .
\end{gathered}
$$

For $t<\phi_{0} / m m_{P l}$ we have

$$
H=H\left(\phi_{0}\right)=\sqrt{\frac{4 \pi}{3}} \frac{m \phi_{0}}{m_{P l}},
$$

and

$$
R(t)=R_{0} \exp \left(H\left(\phi_{0}\right) t\right) .
$$

If the scalar field is initially sufficiently highly excited $\phi_{0}>m_{P l}$ the universe expands exponentially for a long time and $H\left(\phi_{0}\right) t_{\text {final }} \gg 1$ so the scale factor grows by many 
orders of magnitude. During the inflationary stage of evolution of the universe the mean density of matter and its temperature exponentially decrease to zero. All primordial perturbations become smoothed out and the universe quickly approaches the de Sitter stage (cosmic no hair theorem).

Let us consider therefore small perturbations of the flat Friedman cosmological model. Following Stewart and Lyth [9], we write the metric of the perturbed spacetime in the following form

$$
d s^{2}=R^{2}(\eta)\left[d \eta^{2}-\left(\delta_{i j}+2 h_{i j}\right) d x^{i} d x^{j}\right],
$$

and expand the small perturbations $h_{i j}$ into plane waves, so

$$
h_{i j}=\int \frac{d^{3} \vec{k}}{(2 \pi)^{3 / 2}} \sum_{s=1}^{2} \psi_{\vec{k}, s}(\eta) e_{i j}(\vec{k}, s) e^{i \vec{k} \cdot \vec{x}},
$$

where $e_{i j}(\vec{k}, s)$ is the polarization tensor, which satisfies the following normalization and gauge conditions

$$
\begin{gathered}
e_{i j}=e_{j i}, \quad e_{i j} \delta^{i j}=0, \quad k^{i} e_{i j}=0, \\
e^{i j}\left(\vec{k}, s_{1}\right) e_{i j}^{*}\left(\vec{k}, s_{2}\right)=\delta_{s_{1} s_{2}}, \\
e_{i j}(-\vec{k}, s)=e_{i j}^{*}(\vec{k}, s),
\end{gathered}
$$

here $*$ denotes the complex conjugation.

The general action for the gravitational field and the scalar field is

$$
S=-\frac{1}{2} \int \mathcal{R} \sqrt{-g} d^{4} x+\int\left[\frac{1}{2}(\vec{\nabla} \phi)^{2}-V(\phi)\right] \sqrt{-g} d^{4} x,
$$

where $\mathcal{R}$ is the Ricci scalar.

For tensor linear perturbations the action reduces to

$$
\begin{gathered}
S=\frac{1}{2} \int R^{2}\left[\left(h_{i j}^{\prime}\right)^{2}-\left(\partial_{l} h_{i j}\right)^{2}\right] d \eta d^{3} \vec{x}= \\
=\frac{1}{2} \int d^{3} \vec{k} \sum_{s=1}^{2} \int\left[\left|\mu_{\vec{k}, s}^{\prime}\right|^{2}-\left(k^{2}-\frac{R^{\prime \prime}}{R}\right)\left|\mu_{\vec{k}, s}\right|^{2}\right] d \eta,
\end{gathered}
$$

where the prime denotes differentiation with respect to the conformal time $\eta$ and $\mu_{\vec{k}, s}=$ $R \psi_{\vec{k}, s}$.

To describe the spectrum of gravitational waves created during inflation it is useful to quantize the tensor perturbations by introducing creation and annihilation operators. The operator describing an elementary tensor perturbation (a graviton) can be written in the form

$$
\hat{\mu}_{\vec{k}, s}=\mu_{k} \hat{a}_{\vec{k}, s}+\mu_{k}^{*} \hat{a}_{-\vec{k}, s}^{\dagger},
$$

provided that $\hat{a}_{\vec{k}, s}$ satisfy the following commutation relations

$$
\left[\hat{a}_{\vec{k}, s_{1}}, \hat{a}_{\vec{l}, s_{2}}^{\dagger}\right]=\delta_{s_{1} s_{2}} \delta^{(3)}(\vec{k}-\vec{l}),
$$

and

$$
\hat{a}_{\vec{k}, s} \mid 0>=0,
$$


where $\mid 0>$ is the vacuum state and the dagger denotes hermitian conjugation. The equation of motion for $\mu_{k}$ is

$$
\mu_{k}^{\prime \prime}+\left(k^{2}-\frac{R^{\prime \prime}}{R}\right) \mu_{k}=0 .
$$

It is easy to find solutions of this equation in two asymptotic regions: when $R H / k \rightarrow 0$, $\mu_{k} \rightarrow \frac{1}{\sqrt{2 k}} e^{i k \eta}$, and when $R H / k \gg 1, \mu_{k} \sim R$.

Let us note that $R^{\prime \prime} / R$ can be written in the following form

$$
\frac{R^{\prime \prime}}{R}=2 R^{2} H^{2}\left(1-\frac{1}{2} \epsilon\right)
$$

where $\epsilon=-\dot{H} / H^{2}$. The conformal time $\eta$ can be expressed as

$$
\eta=\int \frac{d t}{R(t)}=\int \frac{d R}{R^{2} H}=-\frac{1}{R H}+\int \frac{\epsilon d R}{R^{2} H},
$$

and when $\epsilon=$ const we obtain

$$
\eta=-\frac{1}{R H} \frac{1}{1-\epsilon}
$$

Using (42) we can rewrite equation (40) as

$$
\frac{R^{\prime \prime}}{R}=\frac{1}{\eta^{2}}\left(\mu^{2}-\frac{1}{4}\right),
$$

where $\mu=1 /(1-\epsilon)+1 / 2$.

Substituting (43) into (39) we transform equation (39) into a Bessel type equation and now it is easy to write a particular solution of this equation in the form

$$
\mu_{k}=\frac{\sqrt{\pi}}{2} e^{i\left(\mu+\frac{1}{2}\right) \pi / 2}(-\eta)^{1 / 2} H_{\mu}^{(1)}(-k \eta) .
$$

Asymptotically, for $R H / k \rightarrow \infty$

$$
\mu_{k} \rightarrow e^{i\left(\mu-\frac{1}{2}\right) \pi / 2} 2^{\mu-\frac{3}{2}} \frac{\Gamma(\mu)}{\Gamma\left(\frac{3}{2}\right)} \frac{1}{\sqrt{2 k}}(-k \eta)^{\frac{1}{2}-\mu} .
$$

The power spectrum of primordial background of gravitational waves is defined by

$$
<0\left|\hat{\psi}_{\vec{k}, s} \hat{\psi}_{\vec{l}, \sigma}^{\dagger}\right| 0>=\frac{2 \pi^{2}}{k^{3}} P(k) \delta^{(3)}(\vec{k}-\vec{l}) \delta_{s \sigma} .
$$

Using (45) we finally obtain

$$
P^{1 / 2}(k)=\left.2^{\mu-\frac{3}{2}} \frac{\Gamma(\mu)}{\Gamma\left(\frac{3}{2}\right)}(1-\epsilon)^{\mu-\frac{1}{2}} \frac{H}{2 \pi}\right|_{R H=k} .
$$

As expected, similarly to the density perturbations, the power spectrum of gravitational waves generated during inflation is flat. The spectrum of gravitational waves produced during inflation is shown in Fig. 1.

Inflation ends with a short reheating period. Universe becomes radiation dominated and resumes further evolution according to the Big Bang scenario. During the radiation dominated era $R \sim \eta$, so $R^{\prime \prime}=0$ and the potential term in the equation (24) vanishes. Therefore during the radiation dominated era the spectrum of primordial gravitational waves does not change. The energy density of primordial gravitational waves decreases due to adiabatic "cooling". Shortly before the hydrogen-helium plasma recombines the universe becomes matter dominated and starts to expand faster with $R \sim \eta^{2}$, so now 


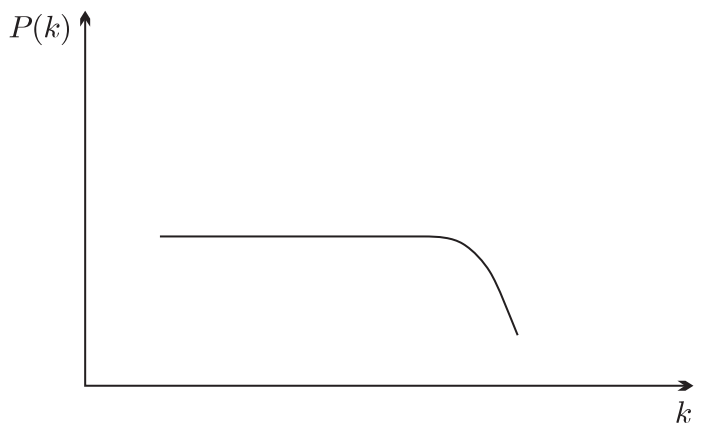

Fig. 1. Schematic representation of spectrum of gravitational waves produced during inflation. The wave vector is drawn on $x$ axis and $\Omega_{g w}$ on $y$ axis.

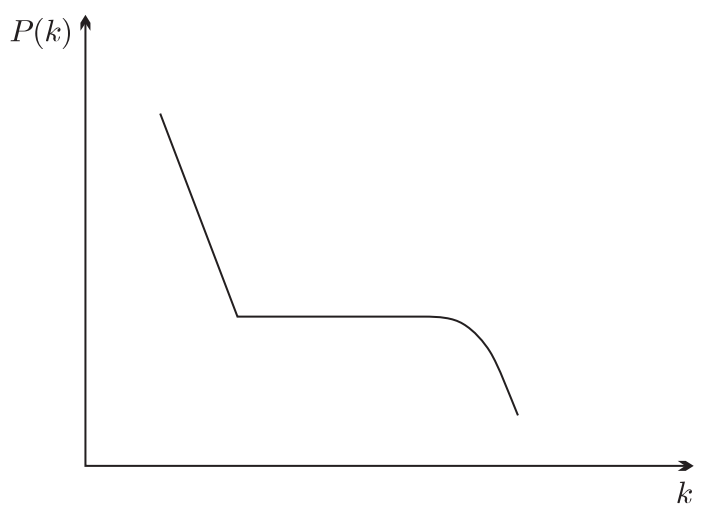

Fig. 2. Schematic representation of expected spectrum of cosmological background of gravitational waves. The wave vector is drawn on $x$ axis and $\Omega_{g w}$ on $y$ axis.

$R^{\prime \prime} / R \sim \eta^{-2}$ and the potential term in the equation (24) becomes different from zero. Characteristic scale determined by the moment of transition from the radiation to the matter dominated epoch of evolution of the universe appears. The amplitude of gravitational waves with wavelengths larger than this characteristic scale becomes amplified and the spectrum of primordial gravitational waves is altered. The final expected spectrum of the cosmological background gravitational waves is shown in Fig. 2.

Only recently reliable limits of the energy density of primordial gravitational waves of very small frequencies have been established. To compare theoretical predictions with observational limits let us recall that the critical energy density is defined as

$$
\rho_{\text {crit }}=\frac{3 c^{2} H_{0}^{2}}{8 \pi G} \approx 1.6 \times 10^{-8} \mathrm{ergs} / \mathrm{cm}^{3},
$$

where $H_{0}$ is the present value of the Hubble constant in $\mathrm{km} / \mathrm{sMpc}$, and $h=H_{0} / 100$. To describe the spectrum of primordial gravitational waves we introduce a dimensionless function of frequency

$$
\Omega_{g w}(f)=\frac{1}{\rho_{\text {crit }}} \frac{d \rho_{g w}}{d \ln f},
$$


where $\rho_{g w}(f)$ is the energy density of primordial gravitational waves of frequency $\mathrm{f}$. Assuming that the observed anisotropy of the microwave background radiation detected by the COBE satellite is produced by cosmological background of gravitational waves we obtain the following restriction on $\Omega_{g w}$ for frequencies in the range $H_{0}<f<30 H_{0}$

$$
\Omega_{g w} h^{2}<7 \times 10^{-11}\left(\frac{H_{0}}{f}\right)^{2} .
$$

Another interesting restriction has been obtained from radio pulsar observations. Several millisecond radio pulsars turned out to be very stable clocks. They have been monitored for almost ten years yielding the following limit on $\Omega_{g w}$ at $10^{-8} \mathrm{~Hz}$

$$
\Omega_{g w}\left(10^{-8} H z\right)<10^{-8} \text {. }
$$

Recently new possibilities of observing the background gravitational waves appeared. Several groups are building gravity wave detectors which will use the new technique of laser interferometry. Four projects LIGO, VIRGO, GEO-600, and TAMA-300 should be completed before the end of the century. More sensitive and space based detectors are planned for the first decade of the next century. From the theoretical estimates of $\Omega_{g w}$ at frequencies accessible to the LIGO, VIRGO, GEO-600, and TAMA-300 detectors it unfortunately follows that none of these detectors will be sufficiently sensitive to observe the cosmological background of gravitational waves if it has been produced by the inflationary mechanism.

However it should be kept in mind that there are other scenarios of the very early evolution of the universe, for example a multidimensional stage or creation of cosmic strings which give different estimates of the expected energy density of the cosmological background of gravitational waves. More details about cosmological background of gravitational waves created in these scenarios can be found, for example in [1], [2], and [3].

\section{References}

[1] B. Allen, The Stochastic Gravity-Wave Background: Sources and Detection, SISSA preprint archive astro-phys 9604033

[2] R. A. Battye and E. P. S. Shellard, Primordial Gravitational Waves: a Probe of the Very Early Universe, SISSA preprint archive astro-phys 9604059

[3] M. Demiański and A. Polanrev, Dynamics of Multidimensional Kaluza-Klein Cosmological Models, Phys. Rev. D41 (1990), 3003.

[4] L. Ford and L. Parker, Phys. Rev. D16 (1977), 1601.

[5] L. P. Grishchuk, Amplification of Gravitational Waves in an Isotropic Universe, ZhETP 67 (1974), 825-838.

[6] L. P. Grishchuk, Gravitational Wave Astronomy, Usp. Ph. Nauk 156 (1988), 297-322.

[7] E. M. Lifshitz, On the Gravitational Stability of the Expanding Universe, ZhETP 16 (1946), 587.

[8] A. Linde, Phys. Lett. B175 (1986), 395.

[9] E. D. Stewart and D. H. Lyth, A more accurate analytic calculation of the spectrum of cosmological perturbations produced during inflation, Phys. Lett. B302 (1993), 171175. 